\title{
Three-dimensional imaging of hemifacial microsomia: a case report
}

\author{
(1) Anwesha Biswas, (1) G. Subhas Babu, (1) Shruthi Hegde, (1) Vidya Ajila, (1) Soundarya Sakthivel \\ Nitte (Deemed to be University), AB Shetty Memorial Institute of Dental Sciences (ABSMIDS), Department of Oral Medicine and \\ Radiology, Mangalore, India
}

\section{Date submitted:}

05.08.2020

Date accepted:

28.09.2020

Online publication date: 15.09.2021

\section{Corresponding Author:}

Shruthi Hegde, MDS., Nitte (Deemed to be University), AB Shetty

Memorial Institute of Dental Sciences (ABSMIDS), Department of Oral Medicine and Radiology, Mangalore, India

drshruthihegde@yahoo.co.in

ORCID:

orcid.org/0000-0002-0744-5593

Keywords: Hemifacial microsomia, cone beam computed tomography, condylar hypoplasia

\begin{abstract}
Craniofacial microsomia or hemifacial microsomia (HFM) is a unique congenital facial deformity causing underdevelopment of the structures derived from first and second pharyngeal arches. The maxilla, mandible, temporomandibular joint, ear, trigeminal and facial nerves are commonly affected. This article presents a case of HFM in a 17-year-old male who was reported with unilateral facial hypoplasia. The diagnosis was confirmed with routine and advanced radiological investigations. Cone beam computed tomography with 3D reconstruction played an important role in determining the diagnosis of HFM.
\end{abstract}

\section{Introduction}

The first and second branchial arches are responsible for the development of various facial structures like the maxilla, mandible, temporomandibular joint (TMJ), zygomatic bone and ear (1). The formation and development of these branchial arches is carried out by various neural crest cells and any damage to these cells can result in abnormalities of various associated facial structures (1). The unilateral underdevelopment of hard and soft tissues, characterized by a wide range of anomalies, is called hemifacial microsomia (HFM). The term HFM was coined by Carl Ferdinand von Arlt in 1881. The first case was reported in 1960 s with developmental defects in aural, oral and mandibular regions (2). HFM is reported to be the second most common developmental craniofacial anomaly with an incidence between 1 in 3000 or 5600 births, after cleft lip and palate (3).

This craniofacial disorder includes the underdevelopment of various skeletal tissues (like the maxilla, mandible, temporal and zygomatic bones) and soft tissues such as the external and middle ear, leading to conductive hearing loss in patient (4). Besides the clinical presentation, radiographic investigations are required to confirm the diagnosis of HFM. Various radiographic investigations have been attempted for a long time to determine the exact diagnosis of this pathology. These include routine investigations like panoramic radiograph, lateral and posterioanterior skull view. Whereas, advanced radiographic investigations like computed tomography, cone beam computed tomography (CBCT), and magnetic resonance 
imaging (MRI) are beneficial for both diagnosis and treatment plan of this pathology. This article presents a case of 17-year-old patient suffering from HFM along with clinical and radiological features highlighted.

\section{Case Presentation}

A 17-year-old male patient was reported to the department of oral medicine and radiology with a chief complaint of facial asymmetry since childhood. It was revealed from his history that the patient had facial asymmetry and difficulty in opening the mouth for past 12 years. He was having sensitivity on taking hot and cold food items. No significant medical history was reported. Family history was noncontributory. No significant changes were present on general physical examination. Extraoral examination revealed a gross facial asymmetry on the right side (Figure 1A). The lips were incompetent. Anotia and ear tags were noticed on the right side (Figure 1B). TMJ examination revealed right side deviation with no tenderness, clicking or crepitus. Mouth opening was $38 \mathrm{~mm}$. On intraoral examination, high arched palate and generalized gingival inflammation was noticed. Hard tissue examination revealed dental caries with mandibular right second premolar, first molar and right and left second molars (Figures 1C, 1D). Malocclusion was observed with crowding in anterior region. Based on clinical features, the patient was provisionally diagnosed as HFM affecting the right side, with a differential diagnosis of Goldenhar syndrome. Panoramic radiograph revealed altered condylar and coronoid morphology on the right side. The condyle and ramus of mandible was also smaller on the right side than left side. The joint space was found to be less prominent on the right of TMJ. Mandibular left third molar was horizontally impacted (Figure 2A). Lateral cephalogram revealed the presence of underdeveloped mandible with a steep mandibular plane (Figure 2B). PA view of the patient showed a prominent facial asymmetry (Figure 2C).

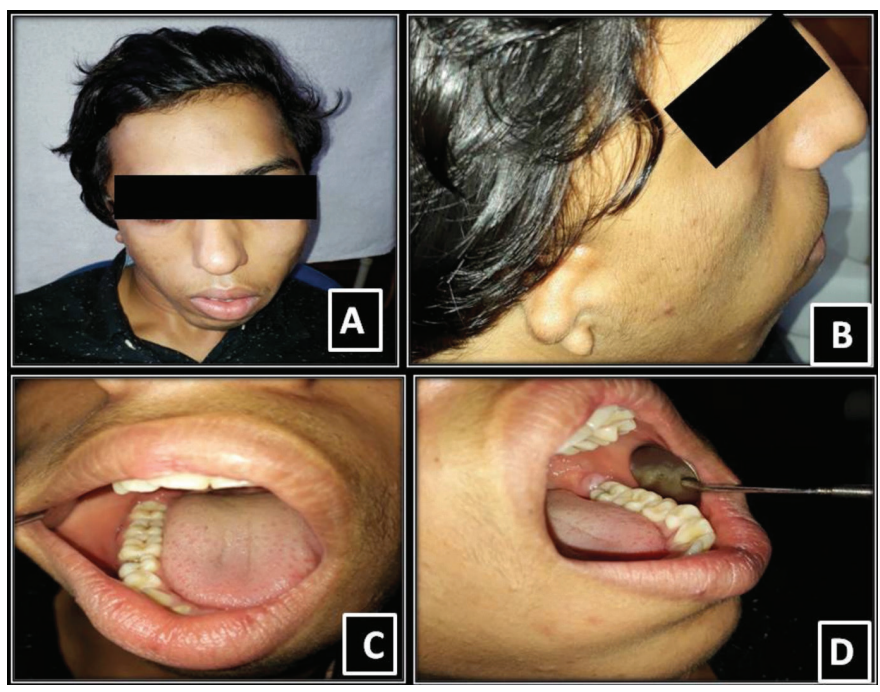

Figure 1. Extra and intraoral examination. A) Extraoral examination revealed gross facial asymmetry on the right side; B) Right ear showing features like anotia and ear tags; C) and D) Dental caries with mandibular right second premolar, first molar and right and left second molars
CBCT images were obtained at various planes. Coronal section revealed that right condyle appeared to be smaller in size than left condyle (Figure $3 \mathrm{~A}$ ). Axial section of CBCT showed morphological alterations on the right side (Figure $3 \mathrm{~B}$ ). In sagittal plane, right condyle was hypoplastic (Figure 3C, 3D). $3 \mathrm{D}$ reconstruction showed asymmetry of the face (Figures $4 \mathrm{~A}$, 4B, 4C). Underdeveloped ear pinna on the right side of face was shown (Figures 4D, 4E).

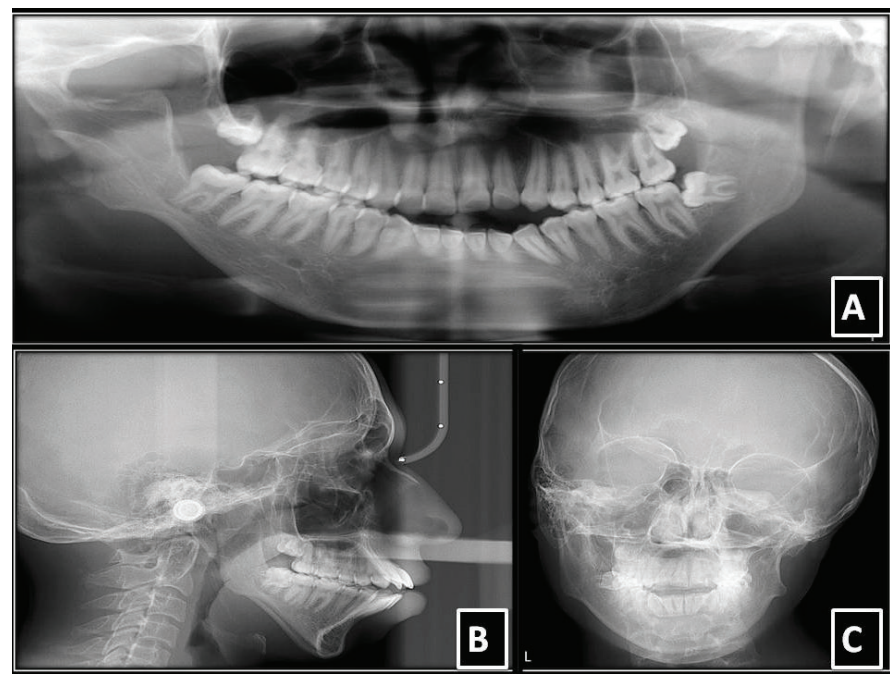

Figure 2. Radiographic examination. A) Panoramic radiograph showing hypoplastic right condyle, reduced ramal height on right side; mesioangular impaction of 38. B) Lateral cephalogram showing the presence of underdeveloped mandible with a steep mandibular plane; C) PA view showed a prominent facial asymmetry

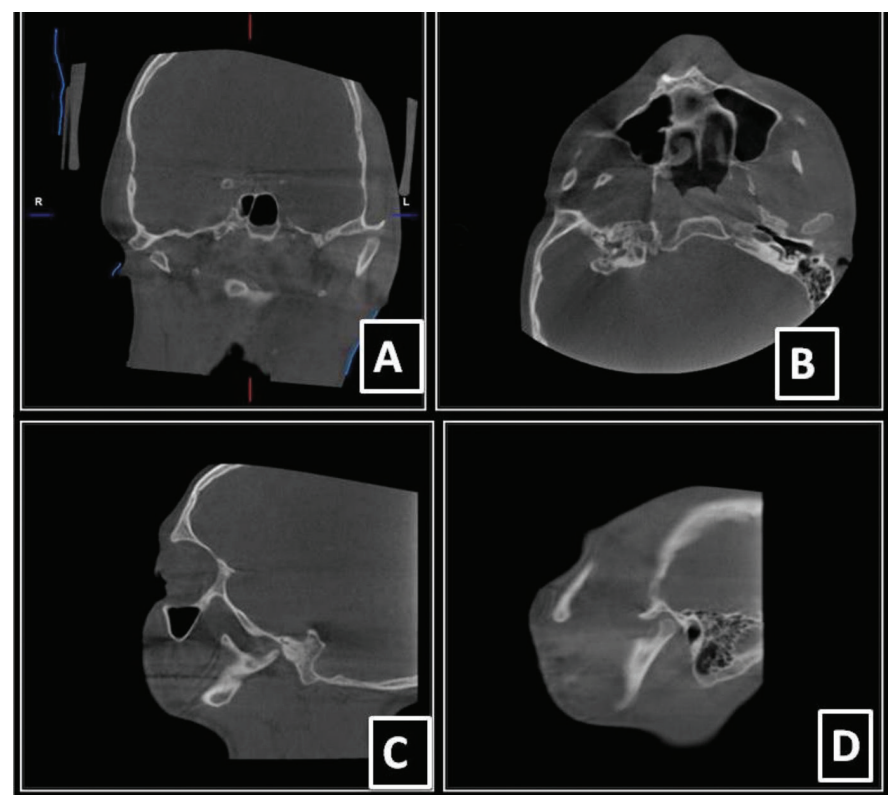

Figure 3. Cone beam computed tomography scan images. A) Coronal view showing altered condylar morphology in the right side; B) Axial view; C and D) Right sagittal view showing hypoplastic condyle compared to left sagittal view 


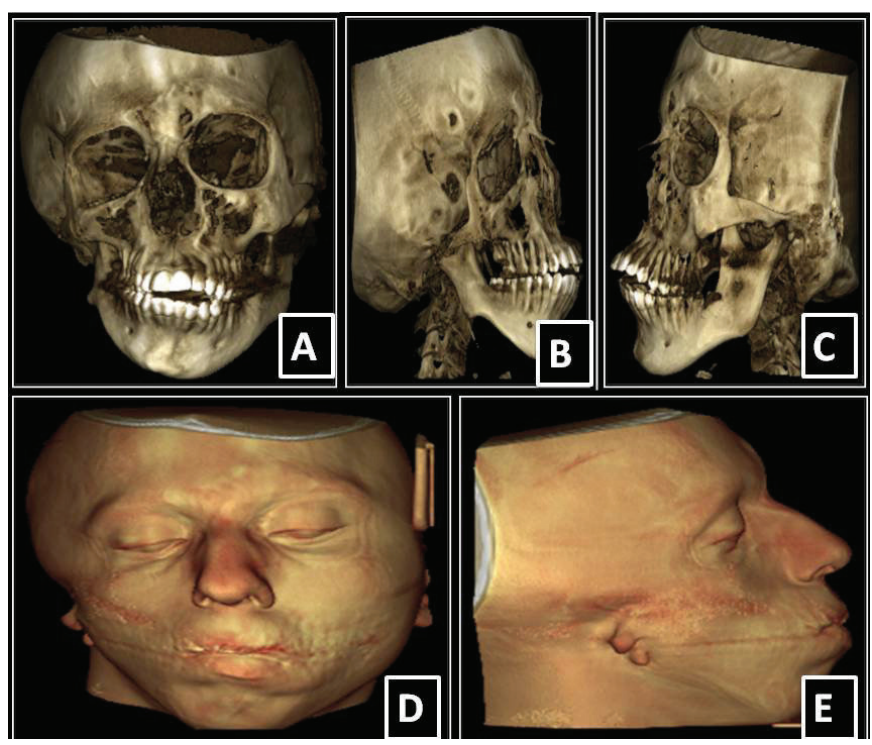

Figure 4. 3D reconstruction shows asymmetry of the face $(A, B, C)$. Underdeveloped ear pinna on the right side of face was shown $(D, E)$

On the basis of clinical and radiographic findings, final diagnosis of HFM was made.

The patient was referred to an otolaryngologist for evaluation. The patient was advised oral prophylaxis and restoration of decayed teeth. The patient was also advised corrective surgery and correction of malocclusion, ear prosthesis for ear abnormalities.

\section{Discussion}

HFM is an anomaly featured by the presence of unilateral hypoplasia affecting various skeletal and soft tissues. It is found that the maxilla, mandible and ear are more commonly affected structures. The disease is characterized by the presence of underdeveloped condyle and coronoid process, short mandibular ramus, malformed ears with preauricular tags (5). The present case also showed similar features. Around 55\% HFM patients were reported with extracranial anomalies affecting the kidney, lungs, heart, skeletal tissues, gastrointestinal and central nervous system.

The etiology of HFM is heterogeneous and unknown. The basis of this disease lies in the disruption of first and second branchial arches during the first 6 weeks of gestation. The disruption could be due to teratogens like retinoic acid, primidone and thalidomide, vascular anomalies and defective genes. Various laboratory studies propose that early loss of neural crest cells is responsible for HFM and its related syndromes (6).

As Meckel's cartilage is responsible for the development of the mandible and middle ear, any damage to Meckel's cartilage in HFM causes abnormality in these skeletal tissues, causing flattening of the affected side of the face (5). Hypoplastic maxillary and mandibular bones, malformed ear, lowering of eye position, and hypoplastic muscles of mastication cause chin and facial midline to appear as shifted to the affected side. Similar clinical presentation was observed in our patient.

Patients with HFM report with mild to severe ear deformities. External ear deformity might range from a flattened helical rim to a complete absence of auricle. Since middle ear ossicles are also derived from first and second branchial arches, thus they are commonly affected in HFM. Stenosis or atresia of the external auditory canal can also be observed. Thus, external and middle ear malformations can cause conductive hearing loss $(7,8)$. The present case had no hearing loss.

Clinical findings are followed by radiographic investigations that include routine and advanced imaging techniques. Routine radiographic investigations revealed hypoplastic maxillary, mandibular and zygomatic bones. Advanced radiographic techniques like CT, CBCT, MRI etc. are required to assess the abnormality in skeletal and soft tissues by observing involved structures in various planes $(9,10)$. CBCT is generally performed in patients of HFM, when facial asymmetry is to be corrected with orthodontic treatment and orthognathic surgery. Furthermore, for orthognathic surgeries, 3D images obtained from CBCT are proven to be very helpful (10). In the present case, we also subjected our patient to undergo $\mathrm{CBCT}$ investigation and $3 \mathrm{D}$ images were also obtained. CBCT scan showed hypoplasia of right side articular eminence, maxilla, mandibular ramus, condyle and coronoid process. External ear structures of the right side were affected.

\section{Conclusion}

HFM is a rare developmental anomaly of the maxillofacial region, which involves one side of the face. For the maintenance of esthetics and proper functioning of the orofacial structures, early treatment intervention is necessary. This can be achieved with proper diagnosis.

\section{Ethics}

Informed Consent: The consent form was filled out by a participant.

Peer-review: Externally peer-reviewed.

\section{Authorship Contributions}

Surgical and Medical Practices: A.B., S.S., Concept: A.B., S.H., Design: A.B., S.H., Data Collection or Processing: A.B., S.H., Analysis or Interpretation: A.B., S.S., Literature Search: S.H., G.S.B., V.A., Writing: A.B., S.H.

Conflict of Interest: No conflict of interest was declared by the authors.

Financial Disclosure: The authors declared that this study received no financial support.

\section{References}

1. Fan WS, Mulliken JB, Padwa BL. An association between hemifacial microsomia and facial clefting. J Oral Maxillofac Surg. 2005;63:330-334. 
2. Ross RB. Lateral facial dysplasia (first and second branchial arch syndrome, hemifacial microsomia). Birth Defects Orig Artic Ser. 1975;11:51-59.

3. Ullal S, Mahale A, Paudel K. Hemifacial microsomia. Indian J Otolaryngol Head Neck Surg. 2008;60:384-386.

4. Kapur R, Kapur R, Sheikh S, Jindal S, Kulkarni S. Hemifacial microsomia: a case report. J Indian Soc Pedod Prev Dent. 2008;(26 Suppl 1):S34-40.

5. Chen Q, Zhao Y, Shen G, Dai J. Etiology and Pathogenesis of Hemifacial Microsomia. J Dent Res. 2018;97:1297-1305.

6. Saddiwal R, Hebbale M, Nisa SU, Sane V. Hemifacial microsomia-A case report and review of literature. Int J Adv Health Sci. 2014;1:9-12.
7. Chaudhari SY. Craniofacial microsomia: A rare case report. J Oral Maxillofac Radiol. 2013;1:70-74.

8. Veliz S, Agurto P, Leiva N. Hemifacial microsomia.Aliterature review. Rev Fac Odontol Univ Antioq. 2016;27:404-424.

9. Birgfeld CB, Saltzman BS, Luquetti DV, Latham K, Starr JR, Heike CL. Comparison of two-dimensional and three-dimensional images for phenotypic assessment of craniofacial microsomia. Cleft Palate Craniofac J. 2013;50:305-314.

10. Choudhary SH, Kale LM, Mishra SS, Swami AN. Hemifacial microsomia: A rare case report. J Indian Acad Oral Med Radiol. 2015;27:603-607. 